\title{
INVARIANT SUBSPACES FOR POSITIVE OPERATORS ACTING ON A BANACH SPACE WITH BASIS
}

\author{
Y. A. ABRAMOVICH, C. D. ALIPRANTIS, AND O. BURKINSHAW
}

(Communicated by Palle E. T. Jorgensen)

\begin{abstract}
Recently we established several invariant subspace theorems for operators acting on an $l_{p}$-space. In this note we extend these results from operators acting on an $l_{p}$-space to operators acting on any Banach space with a (not necessarily unconditional) Schauder basis. For instance, it is shown that if a continuous quasinilpotent operator on a Banach space is positive with respect to the closed cone generated by a basis, then the operator has a nontrivial closed invariant subspace.
\end{abstract}

\section{Preliminaries}

A subset $C$ of a (real or complex) vector space $X$ is said to be a cone whenever $C+C \subseteq C, \alpha C \subseteq C$ for each real $\alpha \geq 0$, and $C \cap(-C)=\{0\}$. Every cone $C$ determines a partial order $\leq$ on $X$ by letting $y \leq x$ whenever $x-y \in C$. The notation $x \geq y$ is, of course, equivalent to $y \leq x$. Thus, the cone satisfies $C=\{x \in X: x \geq 0\}$. The elements of $C$ are known as positive vectors. An ordered vector space is a vector space equipped with a cone $C$. For a detailed account about cones and partially ordered vector spaces, we refer the reader to [4].

In this note the word "operator" will be synonymous with "linear operator". An operator $T: X \rightarrow X$ on an ordered vector space is said to be positive (in symbols $T \geq 0$ or $0 \leq T)$ if $T x \geq 0$ for each $x \geq 0$. For a positive operator $T$, it follows that $T y \leq T x$ whenever $y \leq x$ holds. For operators, the notation $T \geq S$ means $T-S \geq 0$ or equivalently $T x \geq S x$ for each $x \geq 0$.

Recall that a sequence $\left\{x_{n}\right\}$ in a Banach space $X$ is called a Schauder basis (or simply a basis) of $X$ if for every $x \in X$ there exists a unique sequence of scalars $\left\{\alpha_{n}\right\}$ such that $x=\sum_{n=1}^{\infty} \alpha_{n} x_{n}$. Every basis $\left\{x_{n}\right\}$ gives rise to a natural closed cone $C$ defined by

$$
C=\left\{x=\sum_{n=1}^{\infty} \alpha_{n} x_{n}: \alpha_{n} \geq 0 \text { for each } n=1,2, \ldots\right\} .
$$

The cone $C$ will be referred to as the closed cone generated by the basis $\left\{x_{n}\right\}$. For an extensive discussion concerning the cone generated by a basis see [5].

Received by the editors July 23, 1993 and, in revised form, September 27, 1993.

1991 Mathematics Subject Classification. Primary 46A40, 46B40, 47B60, 47B65. 
Associated with every basis is the standard sequence of "coefficient functionals". Let $\left\{x_{n}\right\}$ be a basis of a Banach space $X$. Then the linear functional $f_{n}$ defined by

$$
f_{n}(x)=\alpha_{n} \quad \text { for each } x=\sum_{i=1}^{\infty} \alpha_{i} x_{i}
$$

is a continuous linear functional on $X$. Observe that each $f_{n}$ is also automatically positive with respect to the closed cone generated by the basis $\left\{x_{n}\right\}$. Moreover, the sequence of continuous linear functionals $\left\{f_{n}\right\}$ satisfies $f_{n}\left(x_{m}\right)=\delta_{n m}$.

An operator $T: X \rightarrow X$ on a Banach space with a basis $\left\{x_{n}\right\}$ is said to be positive (with respect to this basis) if $T(C) \subseteq C$, where $C$ is the closed cone generated by $\left\{x_{n}\right\}$. Now fix a basis $\left\{x_{n}\right\}$ for a Banach space $X$. Then every operator $T: X \rightarrow X$ can be identified in the usual manner with an infinite matrix $\left[t_{i j}\right]$. In this context, we can also say that an infinite matrix $\left[t_{i j}\right]$ defines an operator on $X$. Note that an operator $T: X \rightarrow X$ with matrix $\left[t_{i j}\right]$ is a positive operator if and only if $t_{i j} \geq 0$ holds for each pair $(i, j)$. If the basis $\left\{x_{n}\right\}$ is also unconditional, then every positive operator is automatically continuous; see [1, Corollary 2.5, p. 4] or [3, Theorem 12.3, p. 175].

\section{INVARIANT SUBSPACES}

In this section, we shall extend our invariant subspace results for $l_{p}$-spaces to operators acting on a Banach space with a basis. If a basis is specified, then all notions of positivity will always be with respect to the closed cone generated by this basis. As we shall show, the order structure of a Banach space determined by a basis implies some interesting consequences.

Recall that a continuous operator $T: X \rightarrow X$ on a Banach space is said to be quasinilpotent if its spectral radius is zero. It is well known that $T$ is quasinilpotent if and only if $\lim _{n \rightarrow \infty}\left\|T^{n} x\right\|^{1 / n}=0$ for each $x \in X$.

Definition 2.1 ([2]). A continuous operator $T: X \rightarrow X$ on a Banach space is called quasinilpotent at a point $x_{0}$ whenever $\lim _{n \rightarrow \infty}\left\|T^{n} x_{0}\right\|^{1 / n}=0$.

A simple example of a one-to-one positive operator on $l_{1}$ that is quasinilpotent at a positive vector but is not a quasinilpotent operator can be found in [2]. We are now ready to show that on a Banach space with a basis any positive operator that commutes with a positive quasinilpotent operator has a nontrivial closed invariant subspace.

Theorem 2.2. Let $X$ be a Banach space with a basis, and let $T: X \rightarrow X$ be a continuous positive operator. If $T$ commutes with a nonzero positive operator that is quasinilpotent at a nonzero positive vector, then $T$ has a nontrivial closed invariant subspace.

Proof. Let $\left\{x_{n}\right\}$ be a basis of the Banach space $X$, and let $\left\{f_{n}\right\}$ be the sequence of coefficient functionals associated with the basis $\left\{x_{n}\right\}$.

Assume that the nonzero positive operator $A: X \rightarrow X$ satisfies $T A=A T$ and is quasinilpotent at some nonzero positive vector $y_{0}$, i.e., $\lim _{n \rightarrow \infty}\left\|A^{n} y_{0}\right\|^{1 / n}=$ 0 . If $A y_{0}=0$, then the kernel of $A$ is a nontrivial closed subspace that is invariant under $T$. Thus, we can suppose that $A y_{0}$ is nonzero. By an appropriate scaling of $y_{0}$, we can assume that $0 \leq x_{k} \leq y_{0}$ and $A x_{k} \neq 0$ for some $k$. 
Now let $P: X \rightarrow X$ denote the continuous projection onto the vector subspace generated by $x_{k}$ defined by $P(x)=f_{k}(x) x_{k}$. Clearly, $0 \leq P x \leq x$ holds for each $0 \leq x \in X$. We claim that

$$
P T^{m} A x_{k}=0
$$

for each $m \geq 0$. To see this, fix $m \geq 0$ and let $P T^{m} A x_{k}=\alpha x_{k}$ for some nonnegative scalar $\alpha \geq 0$. Since $P$ is a positive operator and the composition of positive operators is a positive operator, it follows that

$$
0 \leq \alpha^{n} x_{k}=\left(P T^{m} A\right)^{n} x_{k} \leq\left(T^{m} A\right)^{n} x_{k}=T^{m n} A^{n} x_{k} \leq T^{m n} A^{n} y_{0} .
$$

Since $f_{k}$ is a positive linear functional, the above inequality yields

$$
0 \leq \alpha^{n}=f_{k}\left(\alpha^{n} x_{k}\right) \leq f_{k}\left(T^{m n} A^{n} y_{0}\right) .
$$

Consequently, $0 \leq \alpha^{n} \leq\left\|f_{k}\right\|\|T\|^{m n} \cdot\left\|A^{n} y_{0}\right\|$, and so

$$
0 \leq \alpha \leq\left\|f_{k}\right\|^{1 / n}\|T\|^{m} \cdot\left\|A^{n} y_{0}\right\|^{1 / n} .
$$

From $\lim _{n \rightarrow \infty}\left\|A^{n} y_{0}\right\|^{1 / n}=0$, we see that $\alpha=0$, and thus condition $(*)$ must hold.

Now consider the subspace $Y$ generated by $\left\{T^{m} A x_{k}: m=0,1, \ldots\right\}$. Clearly, $Y$ is invariant under $T$, and since $0 \neq A x_{k} \in Y$, we see that $Y \neq\{0\}$. Also, for each $y \in Y$, it follows from $(*)$ that

$$
f_{k}(y)=f_{k}(P y)=0,
$$

and consequently $f_{k}(y)=0$ for all $y \in \bar{Y}$. The latter shows that $\bar{Y}$ is a nontrivial closed vector subspace of $X$ that is invariant under the operator $T$, and the proof is complete.

Corollary 2.3. Let $X$ be a Banach space with a basis. If $T: X \rightarrow X$ is a continuous quasinilpotent positive operator, then $T$ has a nontrivial closed invariant subspace.

One can add arbitrary weights to the matrix representing a quasinilpotent positive operator and still be guaranteed that a nontrivial closed invariant subspace exists.

Theorem 2.4. Let $X$ be a Banach space with a basis. Assume that a positive matrix $A=\left[a_{i j}\right]$ defines a continuous operator on $X$ that is quasinilpotent at a nonzero positive vector. If for a double sequence $\left\{b_{i j}\right\}$ of complex numbers the weighted matrix $B=\left[b_{i j} a_{i j}\right]$ defines a continuous operator $B$ on $X$, then the operator $B$ has a nontrivial closed invariant subspace.

Proof. Let $\left\{x_{n}\right\}$ be a basis of the Banach space $X$, and let $\left\{f_{n}\right\}$ be the sequence of coefficient functionals associated with the basis $\left\{x_{n}\right\}$. Assume that the positive operator $A=\left[a_{i j}\right]$ satisfies $\lim _{n \rightarrow \infty}\left\|A^{n} y_{0}\right\|^{1 / n}=0$ for some nonzero positive vector $y_{0} \neq 0$. An appropriate scaling of $y_{0}$ shows that there exists some $k$ satisfying $0 \leq x_{k} \leq y_{0}$. If $A x_{k}=0$, then an easy argument shows that $B x_{k}=0$, and thus the kernel of $B$ is a nontrivial closed invariant subspace (here we assume, of course, that $B \neq 0$ ). Thus, we can suppose that $A x_{k}$ is nonzero.

Now let $P: X \rightarrow X$ denote the positive projection defined by $P(x)=$ $f_{k}(x) x_{k}$. Then arguing as in the proof of Theorem 2.2, we can establish that 
$P A^{m} x_{k}=0$ for each $m \geq 1$. In particular, we have $f_{k}\left(A^{m} x_{k}\right)=0$ for each $m \geq 1$. Consequently, for each $m \geq 1$ and for each positive operator $S: X \rightarrow X$ satisfying $0 \leq S \leq A^{m}$ we have

$$
0 \leq f_{k}\left(S x_{k}\right) \leq f_{k}\left(A^{m} x_{k}\right)=0 .
$$

Next, consider the vector subspace $Y$ generated by the set

$$
\left\{S x_{k}: \exists S \text { such that } 0 \leq S \leq A^{m} \text { for some } m \geq 1\right\} .
$$

Clearly, $Y$ is invariant under each operator $R$ that satisfies $0 \leq R \leq A$. Also, from $(* *)$, it follows that

$$
f_{k}(y)=0
$$

for all $y \in \bar{Y}$. The latter shows that $\bar{Y}$ is a nontrivial closed vector subspace of $X$ that is invariant under each operator $R: X \rightarrow X$ satisfying $0 \leq R \leq A$.

Next, consider the operator $A_{i j}$ defined by $A_{i j}\left(x_{j}\right)=a_{i j} x_{j}$ and $A_{i j}\left(x_{m}\right)=0$ for $m \neq j$. Since the operator satisfies $0 \leq A_{i j} \leq A$, it follows that $\bar{Y}$ is invariant under each of the operator $A_{i j}$. Therefore, the vector subspace $\bar{Y}$ is invariant under the operators

$$
B_{n}=\sum_{i=1}^{n} \sum_{j=1}^{n} b_{i j} A_{i j} .
$$

However, the sequence of operators $\left\{B_{n}\right\}$ converges in the strong operator topology to $B$. Therefore, $B(\bar{Y}) \subset \bar{Y}$ holds, and thus the operator $B$ has a nontrivial closed invariant subspace.

Corollary 2.5. Let $X$ be a Banach space with a basis. Assume that a positive matrix $A=\left[a_{i j}\right]$ defines a continuous operator on $X$ which is quasinilpotent at a nonzero positive vector. If a continuous operator $T: X \rightarrow X$ is defined by a matrix $T=\left[t_{i j}\right]$ satisfying $t_{i j}=0$ whenever $a_{i j}=0$, then the operator $T$ has a nontrivial closed invariant subspace.

We conclude with two remarks.

(1) Consider a quasinilpotent operator on a Banach space with a basis. Suppose the operator is not positive with respect to this basis. At first glance, it appears that our invariant subspace theorems do not apply. However, if one considers a change of basis, then the operator might become positive with respect to the new basis, and therefore, it would have a nontrivial closed invariant subspace. It would be interesting to find out when a given quasinilpotent operator on a Hilbert space can be made positive with respect to some basis.

(2) It is well known that if a Banach space $X$ has an unconditional basis, then (up to an equivalent norm) $X$ is a discrete Banach lattice. Therefore, some of the results obtained in [2] are indeed special cases of the results obtained here.

\section{REFERENCES}

1. Y. A. Abramovich, C. D. Aliprantis, and O. Burkinshaw, Positive operators on Krein spaces, Acta Appl. Math. 28 (1992), 1-22.

2. _ Invariant subspaces of operators on $l_{p}$-spaces, J. Funct. Anal. 115 (1993), 418-424.

3. C. D. Aliprantis and O. Burkinshaw, Positive operators, Academic Press, New York and London, 1985. 
4. A. L. Peressini, Ordered topological vector spaces, Harper and Row, New York and London, 1967.

5. I. Singer, Bases in Banach spaces, Springer-Verlag, Berlin and New York, 1970.

Department of Mathematical Sciences, Indiana University PuRdue University IndiANAPOLIS, INDIANAPOLIS, INDIANA 46202-3216 